\title{
基于 ISM 绿色施工影响因素结构模型的研究
}

\author{
Research on Structural Model of Influencing Factors of Green Construction Based on ISM \\ 于风晓 ${ }^{1}$ 于耀顺 ${ }^{2}$ 马玲岩 ${ }^{1}$ 孙文童 ${ }^{1}$ 姚道洪 ${ }^{1}$ \\ Fengxiao $\mathrm{Yu}^{1} \quad$ Yaoshun $\mathrm{Yu}^{2}$ Lingyan $\mathrm{Ma}^{1} \quad$ Wentong Sun ${ }^{1}$ Daohong $\mathrm{Yao}^{1}$
}

\author{
1. 青岛理工大学 \\ 中国・山东 临沂 273400 ; \\ 2.山东中兴市政集团有限公司 \\ 中国・山东 临沂 276000 \\ 1. Qingdao University of Technology, \\ Linyi, Shandong, 273400, China; \\ 2. Shandong Zhongxing Municipal Group Co.,Ltd., \\ Linyi, Shandong, 276000, China
}

【摘要】绿色建筑施工是工程管理过程中的关键性工作。论文通过查阅文献、专家咨询及 实地走访等方式最终确定影响绿色施工的 12 个因素, 并构建影响绿色施工的解释结构模 型( ISM), 从而明确各影响因素之间的关系。

【Abstract】Green building construction is a key task in the project management process. In this paper, 12 factors affecting green construction are finally determined through literature review, expert consultation and field visits, and an Interpretative Structural Model (ISM) affecting green construction is constructed to clarify the relationship between the various influencing factors.

【关键词】绿色施工; 系统解释结构模型; 影响因素; 工程管理

【Keywords Igreen construction; interpret structural model; influencing factor;

engineering management

【DOI】10.36012/etr.v2i1.1017

\section{1 引言}

近年来, 由于建筑行业在施工的过程中会消耗大量的能 源, 会对自然环境产生巨大的影响。因此, 在建筑施工的过程中 能否做到“四节一环保”成为绿色建筑施工亟待解决的焦点[1], 而影响绿色施工的因素有很多, 而且各个因素之间的关系非 常复杂, 结构之间存在不清晰的问题。因此, 需要建立一套科 学合理的影响绿色施工因素的方法, 以便为绿色建筑施工评 价指标体系的建立提供支持。

目前, 针对绿色施工的研究, 中国学者进行了大量的研究 而且主要集中在绿色施工具体的实施过程方面。马力等 ${ }^{[21 从 ~}$ 可持续发展的角度阐述了绿色施工在工程中的应用问题及对 策; 杨爱歌阳以成本控制为切入点, 节省支出来促进绿色施工 的普及与推广; 郭威东 ${ }^{[4]}$ 主要在绿色建筑施工的质量控制做 研究。这些研究大多注重的是施工的实施过程方面, 而对于影 响绿色施工的本质因素方面研究并不多。因此,基于中国绿色 建筑施工过程的特点, 论文建立一种基于系统解释结构模型 来研究影响绿色施工的因素, 为绿色建筑施工影响因素的分 析提供一种科学严谨的新思路。

\section{2 绿色施工影响因素模型构建}

\section{1 绿色施工影响因素的确定}

绿色建筑施工的影响因素具有彼此之间关系复杂、结构
模糊与不清晰的特点, 而且受到人、料、机、环境等相关因素的 影响。因此, 论文按照科学性的原则, 通过查阅参考文献、实际 工程项目的调查、专家咨询等方式最终选取 12 个影响因素, 分别是: 绿色施工新技术、施工机械装备、施工现场管理技术、 扬尘污染、噪声污染、建筑垃圾、节能措施、节材措施、节地措 施、环境管理机制、居民评价、认证达标率。

\section{2 邻接矩阵的构建}

根据系统解释结构模型的原理, 为了方便地构建模型, 将 影响绿色施工的 12 个因素记为 $S_{i}(i=1,2,3, \cdots, 12)$, 并将各因 素之间的联系构成邻接矩阵 $\boldsymbol{A}$, 邻接矩阵中第 $i$ 行第 $j$ 列的元 素 $a_{j}=1$, 则表明 $S_{i}$ 对 $S_{j}$ 有直接的影响; 若 $a_{j}=0$, 则表明 $S_{i}$ 对 $S_{j}$ 没有直接的影响。

\section{3 可达矩阵的建立}

可达矩阵反映的是某个元素到另外某个元素是否存在可 达的通路。首先在邻接矩阵 $\boldsymbol{A}$ 的基础上加上单位矩阵 $\boldsymbol{E}$ 得到 新的矩阵 $\boldsymbol{A}_{i}=(\boldsymbol{A}+\boldsymbol{E})^{i}$, 并利用 Matlab 软件进行布尔代数运算, 直至 $\boldsymbol{A}_{1} \neq \boldsymbol{A}_{2} \neq \cdots \boldsymbol{A}_{i-1}=\boldsymbol{A}_{i}(i \leqslant n-1)$ 。

\section{4 层级的划分}

首先, 根据 2.3 中建立的可达矩阵对每个元素 $a_{i j}$ 的可达 集 $R\left(S_{i}\right)=\left\{S_{i} \in N \mid a_{i j}=1\right\}$ 、先行集 $Q\left(S_{i}\right)=\left\{S_{i} \in N \mid a_{i j}=1\right\}$ 及共同集 $R\left(S_{i}\right) \cap Q\left(S_{i}\right)$ 进行整理。其中,可达集指的是从元素 $S_{i}$ 出发能 够到达所有的元素的集合并且在可达矩阵中第 $i$ 行元素等于 
1 所在列的元素; 先行集指的是能够到达要素的所有元素的 集合并且在可达矩阵中第 $j$ 列元素等于 1 所在行的元素。其 次, 在此基础之上进行层级的划分,使其满足可达集与先行集 的交集等于可达集的元素 $S_{i}$ 作为结构的顶层元素, 并在可达 矩阵中划去元素 $S_{i}$ 所在的行与列, 接着从可达矩阵中剩余元 素中继续寻找满足条件的元素, 以此类推, 直到找出结构的最 底层元素。经过计算, 共划分为 5 个层级,如表 1 所示。

\section{表 1 绿色施工影响因素的层级划分}

\begin{tabular}{c|c}
\hline 层级 & 元素 \\
\hline$L_{1}$ & $S_{1} 、 S_{2} 、 S_{11} S_{12}$ \\
\hline$L_{2}$ & $S_{4 、} S_{5} 、 S_{6}$ \\
\hline$L_{3}$ & $S_{7} 、 S_{8} 、 S_{9}$ \\
\hline$L_{4}$ & $S_{10}$ \\
\hline$L_{5}$ & $S_{3}$ \\
\hline
\end{tabular}

\section{5 系统解释结构模型的构建}

层级划分结束后, 就会得到相应的骨架矩阵, 从而得到反 映系统的递解结构矩阵, 最终得到绿色施工影响因素解释结 构模型 ${ }^{[5]}$,如图 1 所示。

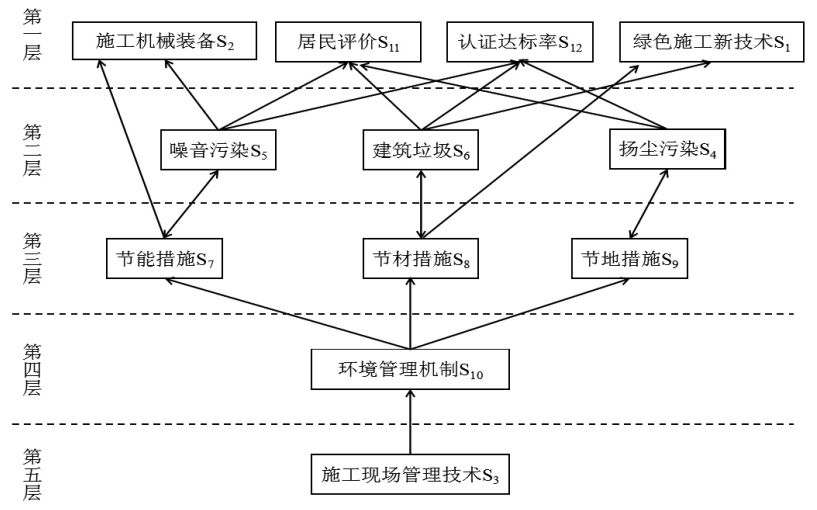

图 1 绿色施工影响因素解释结构模型

\subsection{ISM 模型的分析及建议}

由图 1 可知,影响绿色施工的 12 个因素一共分为 5 个层 级。“施工现场管理技术”位于模型的最底层, 是影响绿色施工 最根本的因素。因为它能够保证工程施工严格按照相关的标 准执行, 有效地减少工程存在的安全隐患问题, 而且能从根本 上解决施工过程中的噪声、扬尘、建筑垃圾等环境方面的问 题。“环境管理机制”位于模型的第四层, 是影响绿色施工的比 较重要的因素。在施工中建设单位与施工单位都承担着绿色 施工的责任，建设单位在招标文件与施工合同中要明确规定 施工单位的环保责任, 施工单位把建设“绿色施工” 的标准运 用到管理体系当中, 因此, 在工程当中要制定相应对策及工程 计划。“施工机械装备”“居民评价”“认证达标率”“绿色施工新 技术”位于模型的最顶层, 是影响绿色施工最直接的因素, 采
用的施工机械直接决定施工过程对环境的影响。比如, 可以采 用低能耗、低噪声的机械为环境做出巨大的贡献; 施工附近居 民的评价可以使工程施工朝着更环保的方向发展；认证达标 率可以使承包商、供应商努力通过 ISO14000 认证。

模型的第二层与第三层是影响绿色施工的间接影响因 素, 各因素之间是强联通块, 可以传递因素之间的关系。在建 筑施工当中产生的噪声主要是由施工机械, 安装及拆卸脚手 架、模板等产生, 可以采用噪声较低、振动频率低的机械并对 施工现场产生的噪声实时监测；扬尘污染是建筑施工中产生 的粉尘、扬尘等悬浮颗粒物造成的空气污染,在作业过程中可 以采取密网覆盖、洒水、硬化路面等措施减少扬尘的产生; 对 于可回收的建筑垃圾实行回收、加工、再利用,对于不可回收 的垃圾按照环保部门的要求统一安排销毁；节材措施可以在 门窗及墙体等维护围护构件、结构材料、装修材料等方面采取 一定措施; 节能措施可以从施工机械、生产及生活临建等方面 入手, 以此达到节约能源的目的; 节地措施需要在施工设计阶 段详细规划, 做到充分合理地布置用地范围。

\section{3 结语}

在建筑工程管理过程中，工程施工会消耗大量的资源与 能源, 而且会对生态环境产生严重的影响, 因此, 实现绿色施 工是生态环境实现可持续发展的重要途径之一。论文通过文 献研究与专家咨询的方式确立影响绿色施工的主要因素, 并 建立起系统解释结构模型从系统的角度对绿色施工影响因素 进行宏观分析,通过分析发现“施工现场管理技术”是影响绿 色施工最重要的因素，“施工机械装备”“居民评价” “认证达标 率” “绿色施工新技术” 是影响绿色施工的直接因素, 并且清楚 地了解了各因素之间的关系，在此基础之上针对各因素对绿 色施工的影响程度提出相关建议及对策。

总体来说, 论文对中国实行绿色建筑施工的实施提出了 切实可行的建议, 具有一定的理论意义与现实价值。

\section{参考文献}

[1]中华人民共和国建设部.绿色施工导则[S].

[2]马力,刘斯鉦,赖晓宇,等.绿色建筑与绿色施工现状及展望 $[\mathrm{J}]$ 内江科技,2019,40(6):109-110.

[3]杨爱歌.建筑工程项目中绿色施工的成本控制研究 [D].泉州: 华侨大学,2017.

[4]郭威东.绿色建筑施工质量控制方法研究[D].兰州: 兰州大学, 2018.

[5]杨伟丽. 基于 ISM 有向图的求可达矩阵的简洁算法 [D].厦门: 厦门大学,2007. 